\title{
GENERALIZED CALIBRATIONS
}

\author{
Jan Gutowski \\ DAMTP, Silver Street, University of Cambridge, Cambridge CB3 9EW \\ jbg21@damtp.cam.ac.uk
}

\begin{abstract}
We present a generalization of calibrations in which the calibration form is not closed. We use this to examine a class of supersymmetric p-brane worldvolume solitons.As an example we consider M5-brane worldvolume solitons in an AdS background.
\end{abstract}

Introduction. There has been considerable progress recently in the classification of p-brane worldvolume solitons. In a flat background with no Born-Infeld type fields the worldvolume dynamics are governed by the Nambu-Goto action

$$
S_{N G}=\int d^{p+1} x \sqrt{-\operatorname{det} g}
$$

where $g$ denotes the pull-back of the background metric to the worldvolume. For such configurations this Lagrangian is equal to the energy density of the p-brane and solutions to the equations of motion minimize both the volume and the energy.

A large number of solutions have been classified in terms of calibrated geometries $[1],[2],[3],[4]$. The solutions are described by calibration forms defined on the target space, and the brane worldvolumes correspond to calibrated sub-manifolds of the target space. Many examples have been constructed, and the types of geometry encountered are typically Kähler, special Lagrangian and exceptional. The resulting solitons may be seen to arise from multiple intersections of p-branes which preserve some proportion of the supersymmetry depending on the geometry of the contact set. However there are limitations to the methods used here, they do not allow treatment of configurations with non-vanishing BornInfeld fields and they do not describe solitons in curved backgrounds 
whose worldvolume actions are modified by the presence of Wess-Zumino terms.

In this article we present a generalization of the concept of a calibrated geometry which enables us to describe a class of solitons in a curved background but with vanishing Born-Infeld fields. It is necessary to modify the definition of the calibration form as in a curved background the energy is not equal to the p-brane volume.

Generalized Calibrations. Let $(M, g)$ be an n-dimensional Riemannian manifold with metric $g$, and $x \in M$. Suppose that $G\left(p, T_{x}(M)\right)$ is the Grassmannian of (oriented) p-planes in $T_{x}(M)$. Then for $\chi \in$ $G\left(p, T_{x}(M)\right)$, there exists an orthonormal basis with respect to g, $\left\{e_{1} \ldots e_{n}\right\}$ of $T_{x}(M)$ such that $\left\{e_{1} \ldots e_{p}\right\}$ is a basis of $\chi$. The co-volume of $\chi$ is then defined as

$$
\vec{\chi}=e_{1} \wedge \ldots \wedge e_{p}
$$

For our definition of a generalized calibration (referred to from now on as simply a calibration), we drop the standard requirement of closure for the calibration form.

A calibration of degree $\mathrm{p}$ on an open subset $U \subset T_{x}(M)$ is a p-form $\phi$ such that, at each $x \in U, \phi_{x}(\vec{\chi}) \leq 1$ for all $\chi \in G\left(p, T_{x}(M)\right)$. It is also required that the contact set $G(\phi)$ should be non-empty, where

$$
G(\phi)=\left\{\chi \in G\left(p, T_{x}(M)\right): \phi(\vec{\chi}) \leq 1\right\} .
$$

Suppose now that $N$ is a p-dimensional submanifold of $M$. Then $N$ is a calibrated submanifold (or calibration for short) of degree $\mathrm{p}$ if

$$
\phi_{x}\left(\vec{N}_{x}\right)=1
$$

for all $x \in N$,where $\phi$ is a calibration of degree $\mathrm{p}$ in $T(M) . \vec{N}_{x}$ is the co-volume of the tangent space $T_{x} N$.

As we have removed the requirement that $d \phi=0$ should hold, it is not the case generally that $N$ is volume minimizing. However another quantity is minimized. Suppose $\mathrm{N}$ is calibrated and $\mathrm{U}$ is an open submanifold of $\mathrm{N}$ and $\mathrm{V}$ is an open sub-manifold of $\mathrm{M}$ such that $\partial U=\partial V$. Then let $L$ be a manifold with oriented boundary $\partial L=U-V$. We may then write

$$
\int \mu_{U}=\int \phi(\vec{U}) \mu_{U}=\int_{U} \phi=\int_{V} \phi+\int_{L} d \phi \leq \int \mu_{V}+\int_{L} d \phi
$$

where we have used Stokes' theorem and $\mu$ denotes the volume form. So the new minimized quantity is

$$
\int d^{p} x \sqrt{\operatorname{det}(g)}-\int_{B} d \phi
$$


where B is a manifold whose boundary is $\mathrm{N}$ and $g$ is the pull-back of the metric to N. This quantity is of considerable interest in theories for which the Born-Infeld fields vanish, and there is a non-trivial WessZumino term; such as p-branes in a curved supergravity background, which we consider in the next section. For these theories, $\mathrm{N}$ is identified with some spatial submanifold of the worldvolume and $\partial B=N$.

Examples. We consider worldvolume solitons on a M5-brane in the background of a stack of parallel M5-branes in the near horizon limit, so the background metric and 4-form are given by

$$
\begin{array}{r}
d s^{2}=\frac{r}{R} d s^{2}\left(\mathbb{E}^{5,1}\right)+\frac{R^{2}}{r^{2}}\left(d r^{2}+r^{2} d s^{2}\left(S^{4}\right)\right) \\
G_{4}=\mu_{S^{4}} .
\end{array}
$$

$R$ is a positive constant and the above geometry is $A d S_{7} \times S^{4}$. We work in the static gauge and consider solutions which depend only on the $5-q$ worldvolume co-ordinates $\left\{x^{i}: i=1, \ldots, 5-q\right\}$. The worldvolume action may then be written as

$$
S=\lambda\left(\int d^{5-q} x \sqrt{\operatorname{det}\left(\tilde{g}_{i j}\right)}-\int \tilde{F}\right)
$$

for constant $\lambda$ and

$$
\tilde{g}_{i j}=\left(\frac{r}{R}\right)^{\frac{q+1}{5-q}}\left(\frac{r}{R} \delta_{i j}+\frac{R^{2}}{r^{2}} \delta_{a b} \partial_{i} y^{a} \partial_{j} y^{b}\right) .
$$

The $y^{a}$ are the transverse scalars. We remark that by adopting an anzatz in which we set $y^{5}=0$ we may set the pull-back of the background 3form to the M5-brane worldvolume to zero so the effective worldvolume action is indeed (1.9). We therefore consider solitons with 2,3 or 4 active transverse scalars.

The definition of the calibration forms proceeds in exactly the same manner as for the flat computations with the co-ordinate basis replaced by an orthonormal basis defined with respect to $\tilde{g}$. Thus for example the $S U(4)$ Kahler calibration generalizes to a $S U(4)$ Hermitian calibration again preserving $\frac{1}{16}$ of the supersymmetry. The calibration form is

$\phi=\frac{r^{3}}{R^{3}} d x^{1} \wedge d x^{2} \wedge d x^{3} \wedge d x^{4}+d x^{1} \wedge d x^{2} \wedge d y^{1} \wedge d y^{2}+d x^{3} \wedge d x^{4} \wedge d y^{1} \wedge d y^{2}$.

It is required that $X^{1}+i X^{2}$ and $X^{3}+i X^{4}$ should be holomorphic functions of $x^{1}+i x^{2}$ and $x^{3}+i x^{4}$. More interesting examples may be obtained 
by considering generalizations of special Lagrangian and exceptional geometries [5]. In all cases it is straightforward to verify that $d \phi=\tilde{F}$ so that the equations of motion are satisfied. All of the examples in this background are supersymmetric, i.e.

$$
\Gamma \epsilon=\epsilon
$$

where for a p-brane with vanishing Born-Infeld fields

$$
\Gamma=\frac{1}{(p+1) !} \epsilon^{\mu_{1} \ldots \mu_{p+1}} \gamma_{\mu_{1}} \ldots \gamma_{\mu_{p+1}}
$$

and $\epsilon$ is a Killing spinor [6]. It has been shown that just as for the flat background the calibration form may be constructed from these Killing spinors satisfying appropriate constraints [7]. Moreover the relation $d \phi=\tilde{F}$ may be seen to arise as a consequence of the supersymmetry algebra.

The methods outlined here may be applied to p-brane configurations in a large number of backgrounds for which the Born-Infeld type fields vanish. In addition, an extension of this treatment has been presented in [8] which includes these fields.

\section{Acknowledgments}

I thank EPSRC for a studentship and the organizers for an excellent school.

\section{References}

[1] R.Harvey and H.B. Lawson, Calibrated Geometries, Acta. Math. 148 (1982) 47.

[2] G.W. Gibbons and G. Papadopoulos, Calibrations and Intersecting Branes, Commun. Math. Phys. 202 (1999) 593.

[3] J.P.Gauntlett, N.D.Lambert and P.C. West, Branes and Calibrated Geometries, Commun. Math. Phys. 202 (1999) 571.

[4] B.S. Acharya, J.M. Figueroa-O'Farrill and B.Spence, Branes at Angles and Calibrations, JHEP 04:012 (1998).

[5] J.B. Gutowski and G.Papadopoulos, AdS Calibrations, hep-th/9902034.

[6] E. Bergshoeff, R. Kallosh, T. Ortín and G. Papadopoulos, $\kappa$-symmetry, Supersymmetry and Intersecting Branes, Nucl. Phys. B502 (1997) 149.

[7] J.B. Gutowski, G.Papadopoulos and P.K. Townsend, Supersymmetry and Generalized Calibrations, hep-th/9905156.

[8] O. Baerwald, N.D. Lambert and P.C. West, A Calibration Bound for the MTheory Fivebrane, hep-th/ 9907170. 